\title{
Analyse de la sûreté des centrales électronucléaires françaises et critères $\left(^{*}\right)$
}

\author{
B. CLÉMENT $(* *)$ \\ (Manuscrit reģu le 17 septembre 1975)
}

\begin{abstract}
RÉSUMÉ
L'objet de cette communication est de présenter les aspects réglementaires et techniques de l'évaluation de la sûreté faite pour les centrales électronucléaires françaises et de faire le point sur les principaux critères de sûreté appliqués ou en cours de préparation. On s'attache à montrer quel est l'apport des critères aux techniques d'évaluation de la sûreté, mais aussi quelles en sont les limites d'emploi et les difficultés à les transposer sans aménagement d'un pays dans un autre.

Un paragraphe particulier traite des caractéristiques des sites nucléaires français et des liaisons existant entre l'évaluation technique de la sûreté des installations et le choix des sites.
\end{abstract}

\begin{abstract}
The technical and regulatory aspects of the French nuclear power plant safety assessment are presented. For each nuclear plant, the assessment is based on fundamental safety principles, using a method taking into account all provisions having an effect on safety. This qualitative method allows probabilistic evaluations to be used. It includes the study of accidents with possible radiological consequences, in order to ensure that, even in these cases, necessary radioprotection actions will be performed.

This assessment work is the basis of safety criteria development and this paper will attempt to describe the status of French criteria, presently applied or being prepared.
\end{abstract}

(*) Communication présentée au $1^{\text {er }}$ Congrès régional asiatique de radioprotection, Bombay, 15-20 décembre 1974.

$\left({ }^{* *}\right)$ Ministère de l'Industrie et de la Recherche, Service Central de Sûreté des Installations Nucléaires, 13, rue de Bourgogne, 75007 Paris.

RADIOPROTECTION, VOL. $10-\mathrm{N}^{\circ} 3$ 


\section{ÉTAPES RÉGLEMENTAIRES DE L'ANALYSE DE SÛRETÉ}

La réglementation française en matière de sûreté nucléaire s'appuie sur le décret du 11 décembre 1963, modifié le 27 mars 1973, relatif aux installations nucléaires de base.

Il appartient au Service Central de Sûreté des Installations Nucléaires placé auprès du Ministère de l'Industrie et de la Recherche de préparer et de mettre en œuvre toutes les actions techniques relatives à la sûreté des installations nucléaires; c'est donc à ce service qu'il incombe, notamment, de mener pour le compte du Ministère l'ensemble des procédures d'autorisation découlant des décrets précités.

Des décisions et des instructions ministérielles précisent les conditions dans lesquelles sont accordées les autorisations nécessaires à la construction et à l'exploitation des installations nucléaires.

Le décret du 11 décembre 1963 modifié prévoit, en particulier, que les installations nucléaires de base, c'est-à-dire les réacteurs, les accélérateurs susceptibles de communiquer aux particules une énergie supérieure à $300 \mathrm{MeV}$, les usines et les installations traitant ou stockant des substances radioactives, ne peuvent être créées qu'après autorisation délivrée par décret après avis d'une Commission interministérielle des installations nucléaires de base et avis conforme du ministre de la Santé. L'autorisation de création fixe les prescriptions techniques que le pétitionnaire est tenu d'appliquer. Ces prescriptions sont établies à l'issue d'un examen technique détaillé fait par le Département de Sûreté Nucléaire du Commissariat à l'Énergie Atomique et présenté par lui devant un Groupe permanent d'experts placé auprès du Service Central de Sûreté des Installations Nucléaires (S. C. S. I. N.).

Le document de base pour l'analyse de sûreté est le rapport préliminaire de sûreté.

Une procédure visant à autoriser les rejets d'effluents radioactifs se déroule simultanément à celle d'autorisation de création. Elle est menée par le S. C. S. I. N., le Ministère de la Santé (Service Central de Protection contre les Rayonnements Ionisants) et le Secrétariat d'État à l'Environnement.

Ensuite, et au moins six mois avant le premier chargement du réacteur, un rapport provisoire de sûreté et les règles générales d'exploitation doivent être remis par le pétitionnaire. Ces documents sont examinés dans les mêmes conditions que le rapport préliminaire.

La dernière phase officielle pour la création et le démarrage d'une centrale est la mise en exploitation normale qui est prononcée après examen du rapport définitif de sûreté qui reprend les éléments des rapports précédents en les complétant et en prenant en compte les enseignements tirés des essais de mise en service.

Il faut noter qu'il n'existe pas de procédures indépendantes d'autorisation pour les sites. Ce sont les installations nucléaires elles-mêmes dont la 
création est autorisée sur un site donné, mais le choix de ceux-ci nécessite des études assez longues et de nombreuses discussions entre les organismes intéressés.

\section{ÉVALUATION TECHNIQUE DE LA SÛRETÉ}

Disposant des informations contenues dans les rapports de sûreté, éventuellement et, si nécessaire, de notes plus détaillées émises par les constructeurs et des renseignements recueillies au cours de nombreux contacts et réunions, l'analyste doit s'assurer, en essayant d'être exhaustif, qu'en fonctionnement normal (régime stable et transitoires normaux) et en cas de défaillance de matériels ou d'erreurs humaines, la protection des personnes et des biens restera assurée.

La méthode utilisée en France a déjà fait l'objet de présentations dans des réunions internationales [1,2]. Elle consiste essentiellement à s'assurer que, pour chacune des barrières disposées entre les produits dangereux et les personnes (c'est-à-dire, généralement, la gaine des éléments combustibles, le circuit primaire et le confinement), les mesures de prévention sont suffisantes, les moyens de surveillance et d'action sont bien adaptés.

Ce type d'analyse permet de faire ressortir le caractère progressif de la sûreté, en distinguant trois étapes successives mais non indépendantes :

- La prévention : la validité de chaque barrière doit être démontrée par le choix des matériaux, leur adaptation aux conditions de fonctionnement et le maintien, dans le temps, des caractéristiques imposées; il est essentiel de mettre en évidence les limites technologiques de manière à définir, en toute connaissance de cause, les marges réelles entre les conditions de fonctionnement et ces limites.

- La surveillance : elle est destinée à détecter toute entrée à l'intérieur des marges précédémment définies afin d'être en mesure, dans ce cas de déclencher à temps une action correctrice, automatique ou manuelle, pour revenir aux conditions normales.

- L'action de sécurité : elle a pour but, en cas de dépassement accidentel des limites technologiques, de prévenir l'émission de produits radioactifs ou d'en limiter l'importance.

Pour chaque barrière cette analyse devra donc se faire comme indiqué précédemment pour les trois cas d'exploitation : fonctionnement normal, transitoires normaux et transitoires accidentels.

La liste des causes de défaillances sera avantageusement établie à partir d'un arbre de fautes mettant en évidence les différents processus pouvant amener la détérioration de la barrière considérée. Lorsque cela est possible, une méthode probabiliste est utilisée pour déterminer la fiabilité de certaines fonctions.

Ces techniques utilisées en fiabilité - arbres de défaillance, arbres de décision, diagrammes logiques - constituent un outil extrêmement utile dans

voL. $10-\mathrm{N}^{\circ} 3$ 
l'analyse de sûreté des diverses barrières. Lorsqu'on examine le cas des transitoires accidentels, elles permettent de vérifier que l'on n'a pas laissé échapper de "chemins critiques" susceptibles de mettre en danger l'intégrité de la barrière; elles mettent en évidence les points faibles de l'installation qui déterminent la fiabilité globale d'un ensemble ou sous-ensemble et auxquels il peut s'avérer nécessaire d'apporter une redondance ou une diversification supplémentaire; elles fournissent les seuls critères objectifs pour le choix entre différentes actions de sécurité.

Cette étude barrière par barrière étant ainsi effectuée et pour évaluer en particulier leur indépendance les unes par rapport aux autres, ce qui est essentiel pour le bilan de sûreté, il est nécessaire d'étudier le déroulement d'accidents types de grande ampleur. Un accident dit " de dimensionnement " est pris en compte pour le calcul des caractéristiques à donner au confinement du réacteur et des équipements nucléaires qui lui sont directement liés. Pour un tel accident, dont la probabilité est très faible compte tenu des précautions prises pour la sûreté de l'installation, les conséquences radiologiques pour les personnes sont calculées et l'on s'assure qu'elles sont acceptables. Ces études permettent une estimation du comportement dynamique des produits radioactifs au cours de leur transfert possible du cœur jusqu'à l'enceinte extérieure, et fournit un ordre de grandeur des conséquences radiologiques pour le site, si l'intégrité de toutes les barrières devait être atteinte. Cette démarche finale ne constitue qu'une part de l'analyse de sûreté, et ne saurait remplacer l'examen par barrière qui permet de mettre en évidence toutes les précautions prises pour éviter de tels accidents, et de souligner les multiples transgressions qu'ils impliqueraient. Elle conserve, cependant, une grande importante pour l'appréciation des qualités du site puisqu'elle doit montrer qu'étant donné l'environnement existant, un accident grave n'aura pas de conséquences inacceptables.

\section{CHOIX DES SITES. CARACTÉRISTIQUES}

Le choix des sites en France ne posait pas, jusqu'à ces dernières années, de problèmes difficiles car le nombre des centrales était faible au regard des possibilités du territoire national. L'accélération du programme français en matière de production d'origine nucléaire fait que 30 à 35 sites nouveaux devront être trouvés d'ici l'an 2000 pour implanter au moins 150 nouveaux réacteurs (puissance prévue, $170 \mathrm{GWe}$ ). Cela donne donc une grande importance aux problèmes soulevés par le choix de ces sites et par l'examen des aspects de sûreté dus à l'interaction des centrales et de l'environnement.

Dès le début du développement des centrales électronucléaires, il a été de pratique courante que les examens de sûreté passent en revue toutes les caractéristiques des sites. Les plans actuellement recommandés pour les rapports de sûreté n'ont fait qu'accentuer cette tendance : population, utilisation des terrains, météorologie, géologie, hydrologie, séismologie et écologie doivent y être présentées. Il est, cependant, de plus en plus insisté sur l'évolution future de l'environnement de la centrale (augmentation de la population, déve- 


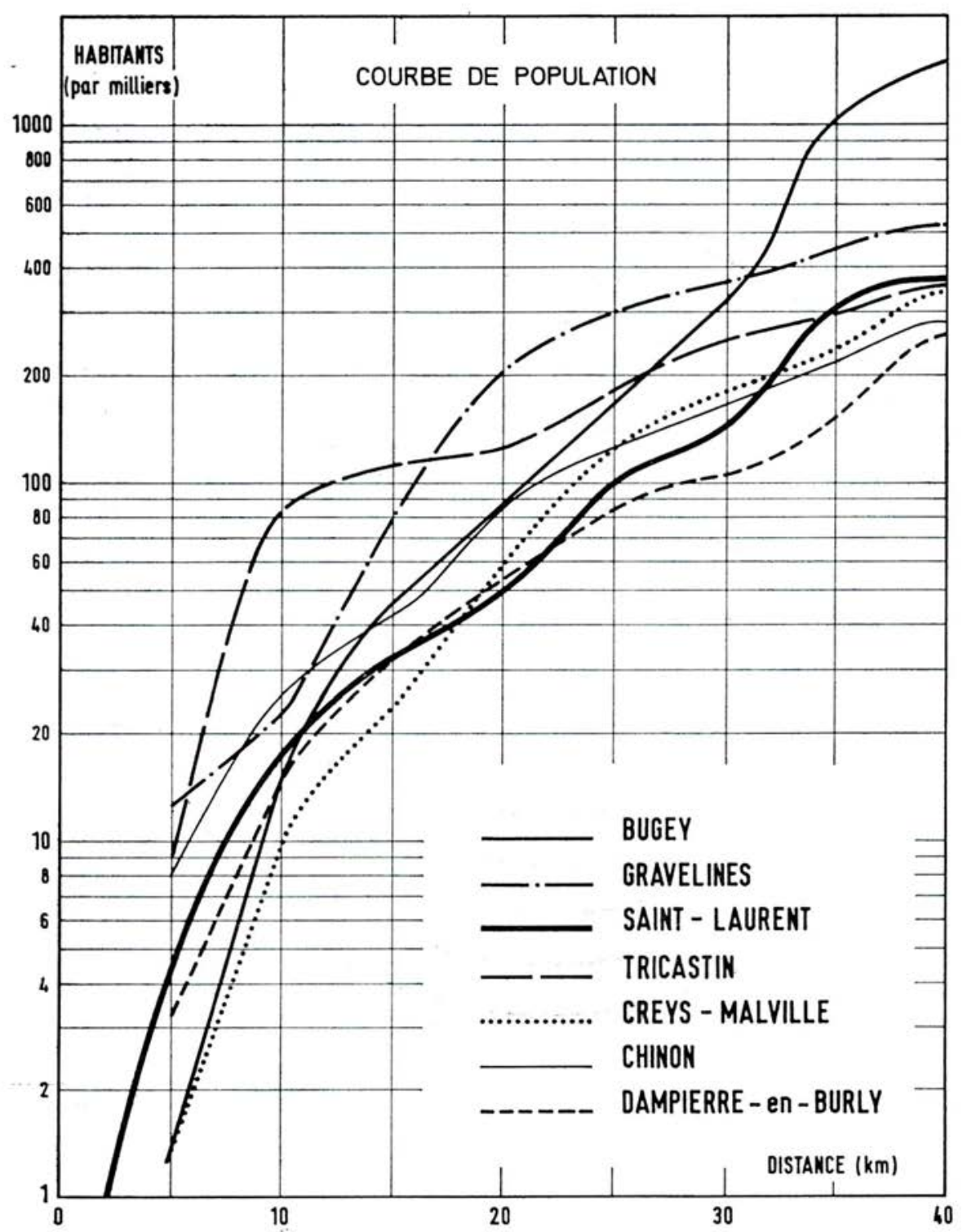

FIG. 1. - Courbe de répartition de la population pour quelques sites en exploitation ou prévus.

vol. $10-\mathrm{N}^{\circ} 3$ 
loppement de zones industrielles, etc.) et sur la nature des activités susceptibles d'être implantées au voisinage. La prévision de sites en bordure de mer et parfois près de ports importants fait que l'on doit examiner de très près les risques que de telles activités peuvent faire courir à la centrale.

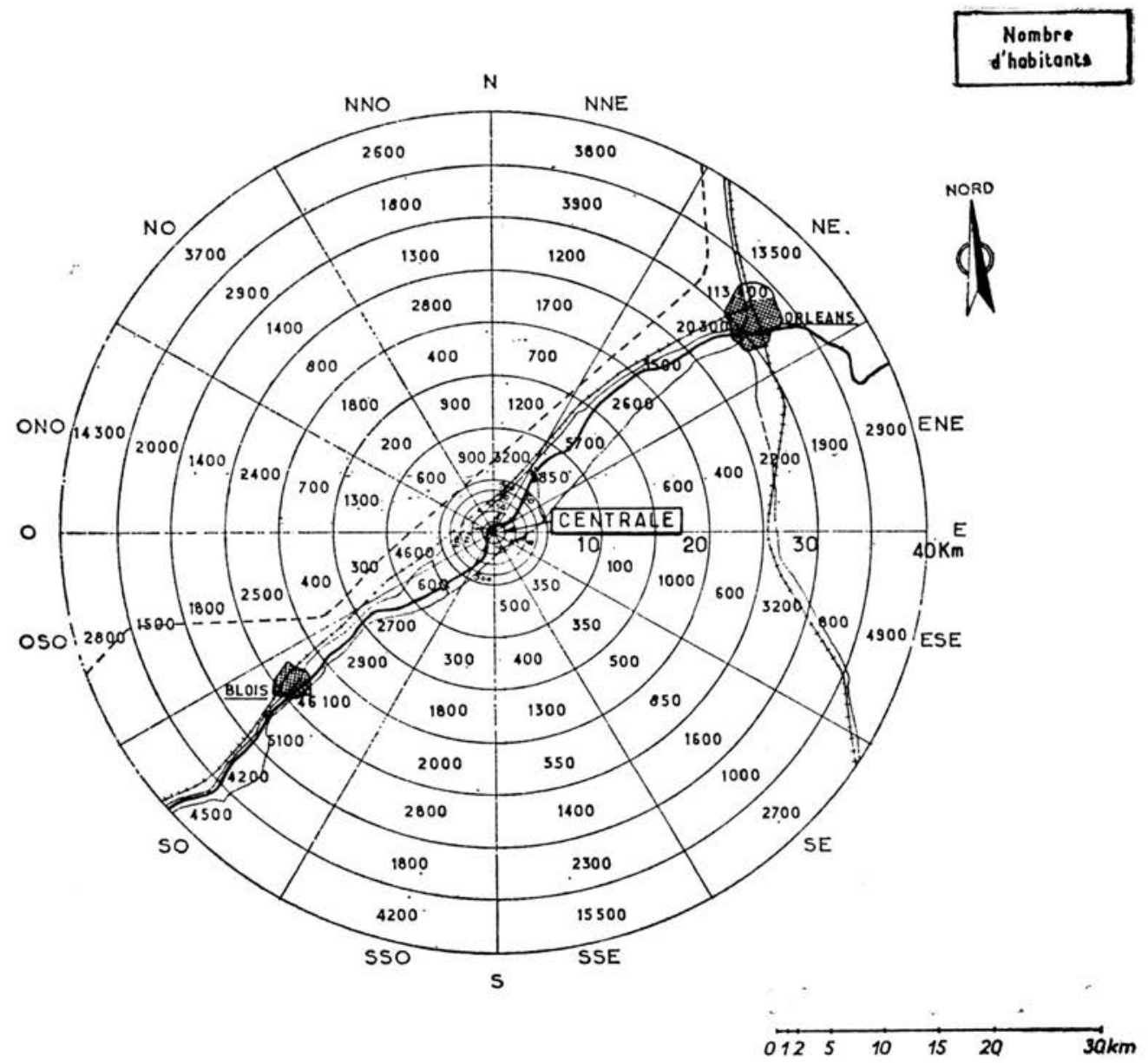

FIG. 2. - Exemple de répartition de la population par secteur.

Les sites actuellement ouverts en France peuvent être considérés par rapport aux sites des autres pays européens et des États-Unis d'Amérique, comme des sites assez satisfaisants. La figure 1 donne une courbe de la répartition des populations pour quelques sites en exploitation ou prévus. 
Le calcul des doses auxquelles le public sera exposé est effectué pour les effluents gazeux et liquides émis en fonctionnement normal et en cas d'accident. Pour ce faire, et pour ce qui concerne les doses dues aux effluents gazeux, la distribution des habitants est établie en divisant l'environnement en secteurs et, à l'intérieur de ces secteurs, en mailles géographiques déterminées par l'azimut et la distance ( fig. 2). Ce découpage est fait généralement jusqu'aux environs de $50 \mathrm{~km}$, sauf si une grande agglomération se trouve un peu plus loin, ce qui incite à étendre la zone étudiée. Pour chacune de ces mailles géographiques, sont calculées les concentrations volumiques moyennes $\left(\mathrm{Ci} / \mathrm{m}^{3}\right)$ pour deux catégories de conditions de diffusion, puis les concentrations intégrées annuelles en tenant compte de la probabilité de persistance des vents. En définitive, on peut ainsi donner la dose moyenne pour les habitants de chaque maille géographique. L'appréciation des doses dues aux effluents liquides est encore actuellement faite d'une façon très pessimiste en calculant la dose annuelle que recevrait un individu utilisant toute l'année comme eau de boisson l'eau du fleuve dans lequel les effluents sont rejetés.

Les études des conséquences radiologiques des accidents sont conduites de la même façon que pour les rejets normaux.

La nécessité d'ouvrir de nombreux sites dans les prochaines années fait que l'on peut souhaiter codifier un peu les critères d'acceptation, ce qui guiderait ceux qui ont la charge de trouver de nouveaux sites et ceux qui doivent faire l'analyse technique de leurs caractéristiques. Des travaux ont été faits en France et dans d'autres pays pour essayer, notamment, de définir des critères de limitation des populations autour des sites. Les communications présentées à des conférences par $\mathrm{P}$. Candes et par R. Le Quinio $[4,5]$, donnent quelques réflexions sur ce sujet et, en particulier, sur la prise en compte de la somme, pour tous les accidents, du produit de leur probabilité par leur conséquence. Pour ces études, sont pris en compte également des accidents plus sévères que ceux considérés pour le dimensionnement des installations (par exemple, non refroidissement du cœur après dépressurisation), mais dont la probabilité est encore beaucoup plus faible. Ces études permettent de définir des facteurs de distance, mais si, comme cela a déjà été montré pour les évaluations techniques de la sûreté des installations, l'association d'une probabilité à la conséquence est une voie intéressante [2-4], elle se heurte à des difficultés lorsqu'on veut chiffrer des probabilités très faibles d'accidents pour les comparer à une probabilité que l'on déclarerait comme acceptable. Une étude d'accident comporte donc l'examen des trois diagrammes qui correspondent respectivement à la perte de la première, de la deuxième et de la troisième barrière, diagrammes qui sont reliés les uns aux autres.

\section{CRITÈRES POUR L'ÉVALUATION TECHNIQUE DE LA SÛRETÉ DES RÉACTEURS}

Quant aux critères techniques particuliers aux installations nucléaires proprement dites, il apparaît que s'ils peuvent aider à concevoir, construire et exploiter des installations sûres, s'ils peuvent faciliter la justification de

vol. $10-\mathrm{N}^{0} 3$ 
cette sûreté par l'exploitant et son évaluation par les autorités responsables, ils ne peuvent permettre de faire l'économie d'une analyse technique détaillée qui, à nos yeux, reste essentielle. De plus, il paraît normal et important que l'établissement de critères soit le résultat de l'expérience acquise au cours des évaluations de sûreté.

Cette expérience est maintenant significative dans notre pays : des centrales graphite-gaz représentant une puissance électrique de $2300 \mathrm{MW}$ fonctionnent très bien depuis plusieurs années, un important programme de construction de centrales à eau ordinaire est en cours (six ou sept nouvelles tranches de 900 ou 1350 MWe pourraient être engagées au cours de chacune des prochaines années), la centrale surrégénératrice Phénix de $250 \mathrm{MWe}$ fonctionne d'une façon très satisfaisante et la procédure d'autorisation pour une centrale à neutrons rapides de $1200 \mathrm{MWe}$ Super-Phénix est en cours. Une nouvelle étape peut donc être désormais franchie pour l'adoption de critères techniques.

Ce recul nécessaire pour établir une réglementation en matière de conception, de construction et d'exploitation des installations n'avait évidemment pas à retarder la publication de normes de radioprotection, qui ont été établies dès le début des travaux en matière nucléaire et qui suivent les recommandations de la Commission Internationale de Protection Radiologique. Des textes complémentaires ont été adoptés en novembre et en décembre 1974; ils précisent les conditions dans lesquelles les rejets d'effluents liquides et gazeux sont désormais autorisés.

L'objectif est donc, maintenant, d'aboutir à une réglementation technique générale, et la première tâche a été de définir le cadre dans lequel tous les textes réglementaires qui seront pris ou qui existent déjà pourront se classer d'une façon cohérente.

Le S. C. S. I. N. a chargé un groupe de travail réunissant des représentants de l'Administration, du Commissariat à l'Énergie Atomique, des constructeurs et des exploitants, de lui présenter des propositions sur ce point.

Ce cadre s'articule en trois niveaux :

- Niveau 1 :

- Principes fondamentaux de sûreté.

- Annexe : terminologie.

- Niveau 2 :

2.1. Critères généraux de sûreté :

- communs à toutes les filières,

- propres à chaque filière.

2.2. Procédures administratives.

- Niveau 3 :

- Prescriptions techniques particulières :

- relatives aux critères généraux,

- relatives aux fonctions principales des réacteurs,

- relatives aux différents composants des réacteurs. 
Quelques remarques concernant le contenu du cadre :

\section{- Niveau 1 :}

Toute réglementation est faite pour satisfaire des principes fondamentaux et il paraît logique d'expliciter les principes qui seront à la base des critères de sûreté.

Indépendamment du principe de protection contre les rayonnements, on précisera des principes :

- de conception : dispositions réalisant une progressivité de la sûreté basée sur des mesures préventives, des moyens de surveillance et des moyens d'action;

- d'organisation de la qualité;

- d'acceptation des risques (on notera ici qu'une situation accidentelle doit être d'autant plus improbable que les dommages qui peuvent en résulter sont susceptibles d'être graves; un tel principe peut déboucher sur un critère probabiliste, mais il ne sera pas fixé dans un proche avenir de valeurs quantitatives);

- d'acceptation d'un site (prise en compte des interactions possibles entre l'installation et l'environnement et cela, compte tenu de prévisions d'évolution de l'environnement pendant toute la vie de l'installation; adaptation des moyens réglementaires pour prendre en compte toute modification significative par rapport aux prévisions);

- définissant les obligations de l'exploitant et des constructeurs.

\section{- Niveau 2 :}

A partir des principes fondamentaux, il s'agit de définir, pour l'exploitant et les constructeurs, les objectifs qu'ils devront atteindre pour obtenir le degré de sûreté jugé nécessaire.

On trouvera des critères d'acceptation des sites, de conception, d'assurance de qualité et des critères de mise en service et d'exploitation. A ce niveau, on se propose, d'abord, d'avoir des critères généraux valables pour toutes les filières, puis ensuite des critères concernant les techniques spécifiques à chaque filière.

\section{- Niveau 3 :}

On trouvera à ce niveau des documents définissant, dans la mesure du possible, des moyens d'atteindre les objectifs fixés par les critères généraux.

Les textes relatifs aux critères généraux traiteront des risques particuliers (séisme, incendie), des hypothèses à retenir pour le calcul des accidents limites, des recommandations relatives à la solution des problèmes particuliers, du contenu détaillé des rapports de sûreté, etc.

Les textes relatifs aux fonctions principales du réacteur seront classés suivant l'ordre adopté dans les rapports de sûreté. Enfin, ceux relatifs aux composants pourraient concerner les cuves, les calculateurs, etc.; c'est à ce niveau que l'on trouvera classé un arrêté ministériel du 15 juin 1970 qui 
réglemente la conception, la construction et l'utilisation des caissons en béton précontraint, et un arrêté en date du 26 février 1974 relatif au circuit primaire principal des chaudières à eau.

Ces documents tiennent compte du contexte administratif, juridique et industriel français. Il s'agit là, en fait, d'une caractéristique générale aux réglementations techniques nationales. Il est, en effet, normal que des critères nationaux soient adaptés aux habitudes de l'industrie, à la doctrine des organismes officiels et tiennent compte de la réglementation préexistante. Cela implique que l'utilisation dans un pays tiers de critères mis au point dans un autre pays se fasse en tenant compte du contexte local et avec une bonne compréhension des idées qui ont présidé à la rédaction.

Une dernière remarque est en rapport avec le caractère évolutif des techniques nucléaires. Les bonnes solutions sont multiples; certaines, tout aussi bonnes ou meilleures que celles appliquées aujourd'hui, seront probablement appliquées dans les années à venir. Des critères de sûreté doivent être un moyen pour maintenir un haut niveau de sûreté aux installations, ils ne doivent pas risquer de bloquer l'évolution de la technique.

\section{BIBLIOGRAPHIE}

[1] Bourgeois J. Évolution des idées françaises sur la sûreté des réacteurs. $4^{\mathrm{e}}$ Conférence, internationale sur l'utilisation de l'énergie atomique à des fins pacifiques, Genève, 6-11 septembre 1971 (A/Conf. 49/P/579) New York, Nations Unies, 1972, 3, 163.

[2] BouRGeors J. L'analyse de sûreté des réacteurs de puissance en France, principes généraux et applications pratiques. Principles and standards of reactor safety, Jülich, 5-9 february 1973, Vienne, AIEA, 1973, 147-166.

[3] États-Unis. USAEC. An assessment of accident risks in U. S. commercial nuclear power plants (draft). USAEC report WASH-1400, 1974.

[4] CANDES P. Pratique et expérience de la sûreté des sites nucléaires en France. ANS topical meeting on nuclear plant siting, Portland, Oregon, Aug. 25-28, 1974.

[5] Le Quinio R. Influence de la puissance et de la distance sur les risques présentés par un réacteur nucléaire. Facteur atmosphérique de site. Population dose evaluation and standards for man and his environment, Portoroz, 20-24 May 1974. Vienne, AIEA, 1974, 83-94. 